\title{
Ventilatory limitations to exercise in CF are dependent on lung function and equation selection
}

T. Kent, J. Trott, C.A. Williams, P.J. Oades, N.J. Withers \& O.W. Tomlinson

1. Royal Devon \& Exeter NHS Foundation Trust Hospital, Exeter, EX2 5DW

2. Children's Health and Exercise Research Centre, Sport and Health Sciences, University of Exeter, EX1 2LU

\section{Objective}

During cardiopulmonary exercise testing (CPET), maximal minute ventilation (VEmax) is closely analysed to determine if a patient is able to achieve maximal effort when ventilatory limited (VL) .VL is determined if the patient's VEmax exceeds $85 \%$ of their predicted maximal voluntary ventilation $(M V V)$, with a number of equations used to predict MVV based on lung function. The aim of this study was to identify how patients of differing disease severity were deemed to have VL when performing a CPET, dependent on how MVV was calculated.

\section{Method}

Eighty-seven patients (56 adults/31 paediatrics, 51 male/36 female) were categorised into severe ( $<40 \% \mathrm{FEV}_{1} \%$ pred, $\mathrm{n}=5,5$ adults), moderate (40-69\% FEV 1 \%pred, $\mathrm{n}=26,22$ adults) and mild $(>70 \%$ $\mathrm{FEV}_{1} \%$ Pred, $\mathrm{n}=56,29$ adults) disease severity. All performed a CPET using a validated combined ramp and supra-maximal verification protocol. VL was determined from three different equations to predict MVV: FEV 1 × 35; FEV 1 × 40; and a paediatric-specific equation $\left(27.7\left(\mathrm{FEV}_{1}\right)+8.8\left(\right.\right.$ PredFEV $\left.\left._{1}\right)\right)$ from Stein et al (2003).

\section{$\underline{\text { Results }}$}

Five $(100 \%)$ of the adult patients in the severe category were deemed VL using both the FEV 1 × 35 $(124.4 \pm 12.2 \%)$ and $\mathrm{FEV}_{1} \times 40(108.8 \pm 10.6 \%)$ equations. In the moderate category, 17 (81\%) adult patients, were VL using the $\mathrm{FEV}_{1} \times 35(109.0 \pm 27.5 \%)$ compared with, 14 (69\%) when using the $\mathrm{FEV}_{1}$ x $40(95.3 \pm 24.0 \%)$ equation. In the mild category, $16(52 \%)$ adults were VL using FEV 1 x 35 (90.6 \pm $19.9 \%)$ compared with 10 (36\%) when using $\mathrm{FEV}_{1} \times 40$ (79.3 $\left.\pm 14.4 \%\right)$. For children using the paediatric (Stein) equation, $3(75 \%)$ of the paediatric patients were VL in the moderate category $(87.2 \pm 2.7 \%)$, compared with $11(41 \%)(83.4 \pm 18.5 \%)$ in the mild category.

\section{Conclusion}

When using both equations within the adult population, it is evident the results differ in determining which patients are VL during a CPET. This study shows there is a need for Standardisation when predicting MVV. 